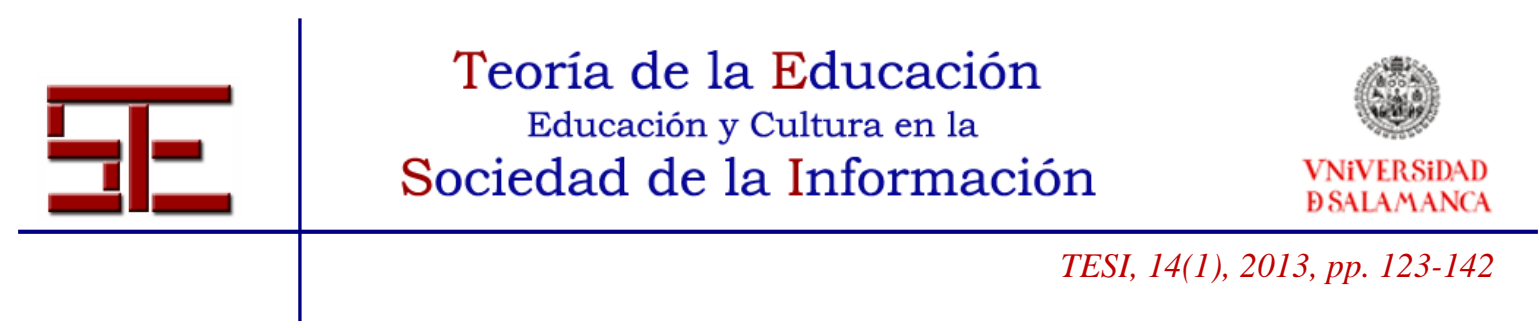

\title{
DESARROLLO DE LAS COMPETENCIAS DE COLABORACIÓN CON EL USO DEL SERIOUS GAME METAVALS
}

Resumen: En el marco de una educación orientada a competencias, el desarrollo de las competencias de colaboración es uno de los principales objetivos de los sistemas educativos actuales. Estas competencias incluyen tanto la comunicación como las diferentes habilidades sociales. La educación orientada a competencias requiere metodologías de aprendizaje activo para poder aplicar el conocimiento en contextos específicos. Entre estas metodologías focalizaremos nuestro interés en el uso de juegos educativos digitales o Serious Games (SG) y en el Aprendizaje Basado en Juegos (ABJ). Los SG han sido analizados como herramientas innovadoras que permiten dar soporte al desarrollo de las competencias de colaboración. Pese a ello, en la orientación curricular actual, los SG tienen una orientación curricular más basada en los contenidos que en el desarrollo de competencias. El objetivo de este estudio es analizar el potencial de los SG para el desarrollo de las competencias de colaboración, a través del análisis del caso de estudio del uso del SG MetaVals.

Palabras clave: Competencias; Colaboración; Habilidades sociales; Aprendizaje Basado en Juegos; Juegos Educativos Digitales; Serious Games.

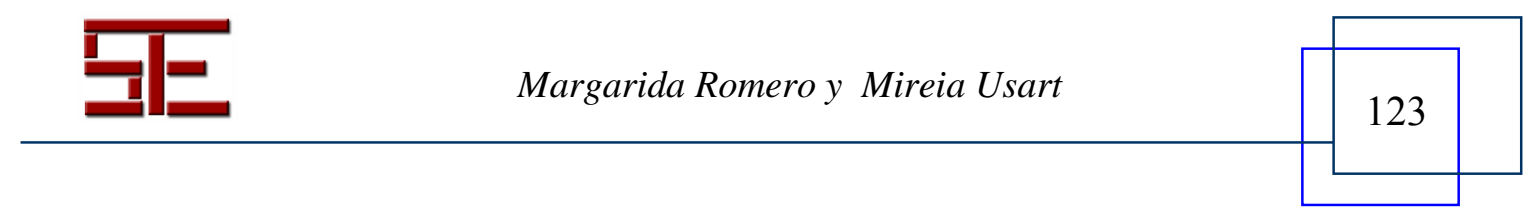




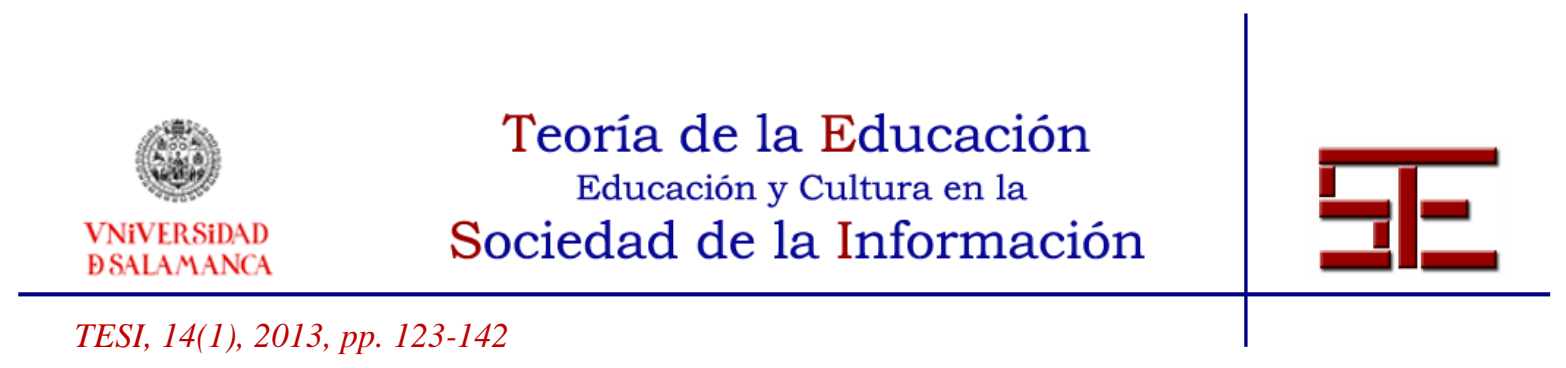

\title{
DEVELOPING THE COLLABORATION SKILLS USING THE SERIOUS GAME METAVALS
}

\begin{abstract}
Developing collaboration skills is one of the main objectives of current competence-oriented educational systems. This development relates also to the communication and social skills. Because of their practice-based orientation, skills and competences require active learning methodologies to be developed, such the use of Serious Games (SG) in Game Based Learning (GBL) methodologies. SGs have been analysed as innovative tools allowing supporting the collaboration skills, although knowledge and curriculum orientation is still dominating the pedagogical research on the use of SG in formal educational contexts. This paper aims to analyse the impact of SG in the collaboration skills, through the analysis of a specific case study focused on the MetaVals SG.
\end{abstract}

Key words: Competencies; Skills; Collaboration; Social skills; Game Based Learning; Serious Games.

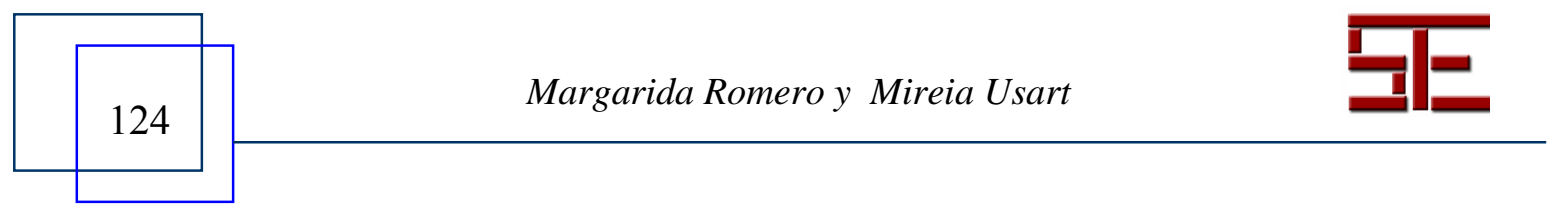




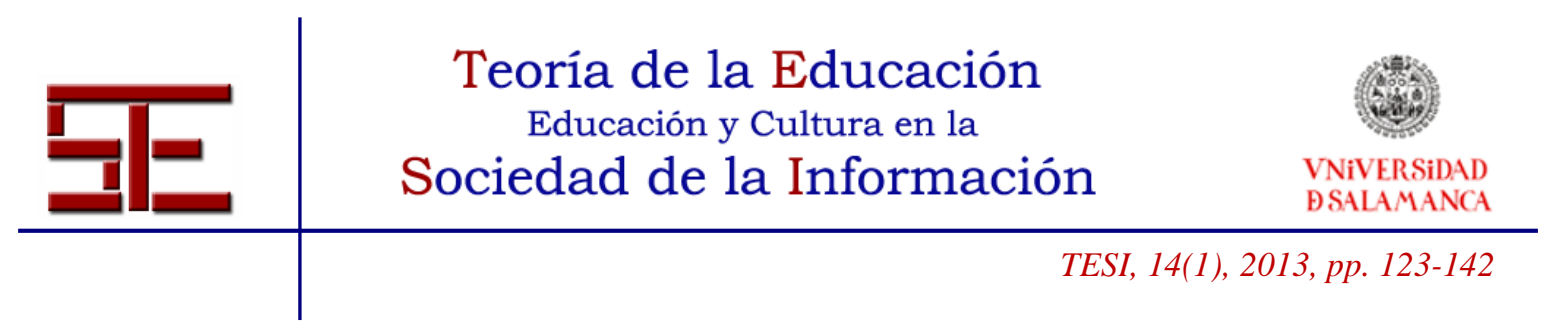

\section{DESARROLLO DE LAS COMPETENCIAS DE COLABORACIÓN CON EL USO DEL SERIOUS GAME METAVALS}

Fecha de recepción: 15/11/2012; fecha de aceptación: 06/01/2013; fecha de publicación: 28/02/2013

Margarida Romero

margarida.romero@esade.edu

ESADE

Mireia Usart Rodríguez
mireia.usart@esade.edu

ESADE

\section{1.- INTRODUCCIÓN}

La sociedad del conocimiento requiere estudiantes más competentes que puedan adaptarse a las profesiones y puestos de trabajo de la sociedad actual y del futuro (Fisch y McLeod, 2009). Este hecho propicia la necesidad de desarrollar las competencias de los estudiantes que les permitan afrontar las necesidades de la sociedad actual y futura en términos de innovación y creatividad, adaptación a los nuevos entornos tecnológicos y en la capacidad a colaborar en entornos de diversidad profesional e intercultural. Este reto educativo conlleva un cambio educativo sustancial que debe convertir los centros educativos en comunidades de aprendizaje (Barrio de la Puente, 2005) y permitir definir un marco competencial para la educación del ciudadano del siglo XXI. Para definir el conjunto de competencias que deben ser consideradas por los sistemas educativos, algunos autores han desarrollado marcos competenciales que han sido denominados bajo el nombre de "competencias del siglo XXI" (Binkley et al, 2010; Dede, 2010; Redecker et al, 2011). Este conjunto de competencias debe permitir a los ciudadanos desarrollarse en sus actividades formativas, profesionales y sociales del siglo XXI (Trilling y Fadel, 2009). Desde los primeros estudios que citan las competencias del siglo XXI (Jones, 1996), ha habido numerosos intentos de identificar y clasificar estas competencias, sin haber llegado aún a un consenso definitivo sobre el conjunto que conforman las competencias del siglo XXI.

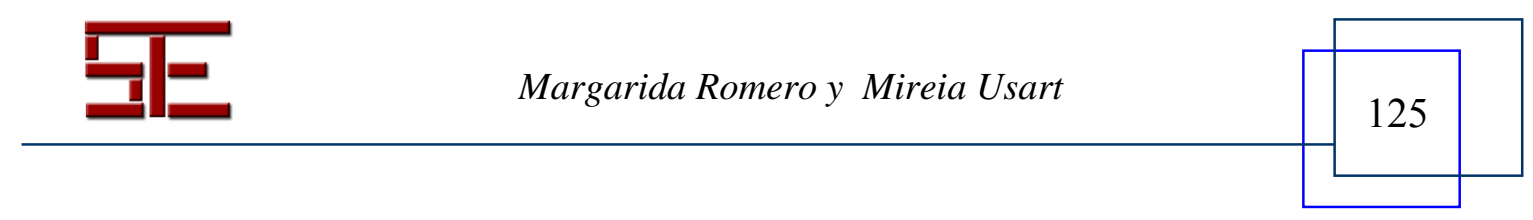




Teoria de la Educación
Educación y Cultura en la
$\begin{gathered}\text { VNiVERSIDAD } \\ \text { BSALAMANCA }\end{gathered}$

Sin embargo, algunos autores han propuesto marcos integradores a partir del análisis de estudios previos que, por regla general, se centran en las competencias siguientes: la capacidad innovadora, la solución creativa de problemas, las competencias metacognitivas, las competencias de comunicación y las competencias relacionadas con la colaboración (Dede, 2010; Redecker et al, 2011). Simultáneamente, las instituciones educativas también han desarrollado y siguen trabajando (Monereo y Pozo, 2007) en la definición de marcos estructurados para identificar las competencias necesarias para el siglo XXI, con el objetivo de organizar estas competencias en un marco conceptual que esté basado en las necesidades educativas actuales y en las necesidades específicas de cada contexto. Para establecer las competencias del siglo XXI relativas a la colaboración, hemos realizado una revisión de la literatura científica sobre las competencias del siglo XXI y los distintos marcos integradores (ATCS, EnGauge, EU, NETS/ISTE, OCDE, P21). Tras el análisis de los diferentes marcos, nos centraremos en el metanálisis de las competencias identificadas por Voogt y Pareja Roblin (2010). Esta categorización integra los principales marcos competenciales anteriores y puede ser considerada como la revisión más completa en este ámbito. Presentamos este listado en la Tabla 1, el cual ofrece una visión general del nivel de frecuencia de cada competencia en los distintos marcos.

Tabla 1. Resumen de las competencias del siglo XXI mencionadas en los seis marcos integradores

\begin{tabular}{|c|c|c|c|}
\hline $\begin{array}{l}\text { Mencionadas en todos } \\
\text { los marcos }\end{array}$ & $\begin{array}{l}\text { Mencionadas en la } \\
\text { mayoría de los } \\
\text { marcos (p. ej. P21, } \\
\text { EnGauge, ATCS y } \\
\text { NETS/ISTE) }\end{array}$ & $\begin{array}{l}\text { Mencionadas } \\
\text { pocos marcos }\end{array}$ & $\begin{array}{l}\text { Mencionadas sólo en } \\
\text { uno de los marcos }\end{array}$ \\
\hline $\begin{array}{l}\text { - Colaboración } \\
\text { - Comunicación } \\
\text { - Conocimiento de } \\
\text { las TIC } \\
\text { - Competencias } \\
\begin{array}{l}\text { sociales y/o } \\
\text { culturales, } \\
\text { ciudadanía }\end{array}\end{array}$ & 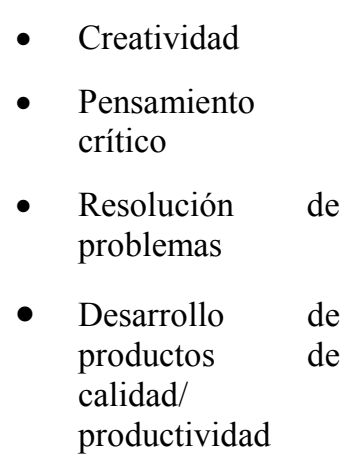 & 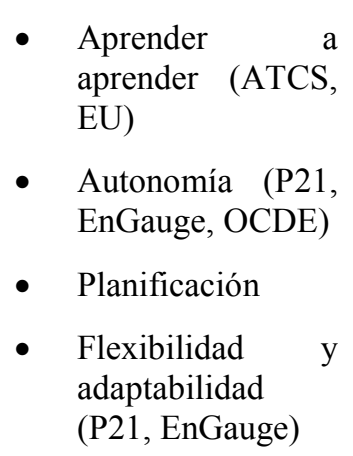 & $\begin{array}{ll}\text { - } & \begin{array}{l}\text { Tomar riesgos } \\
\text { (EnGauge) }\end{array} \\
\text { - } & \begin{array}{l}\text { Resolución de } \\
\text { conflictos (OCDE) }\end{array} \\
\text { - } & \begin{array}{l}\text { Iniciativa y espíritu } \\
\text { emprendedor. }\end{array} \\
\text { - } & \text { Interdisciplinariedad } \\
& (\mathrm{P} 21)\end{array}$ \\
\hline
\end{tabular}

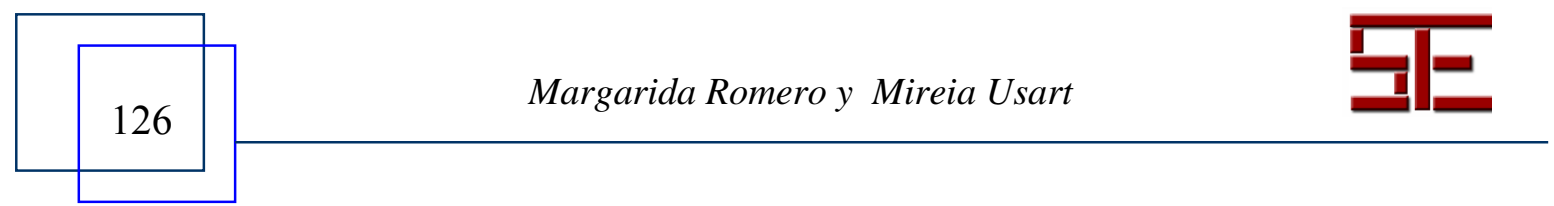



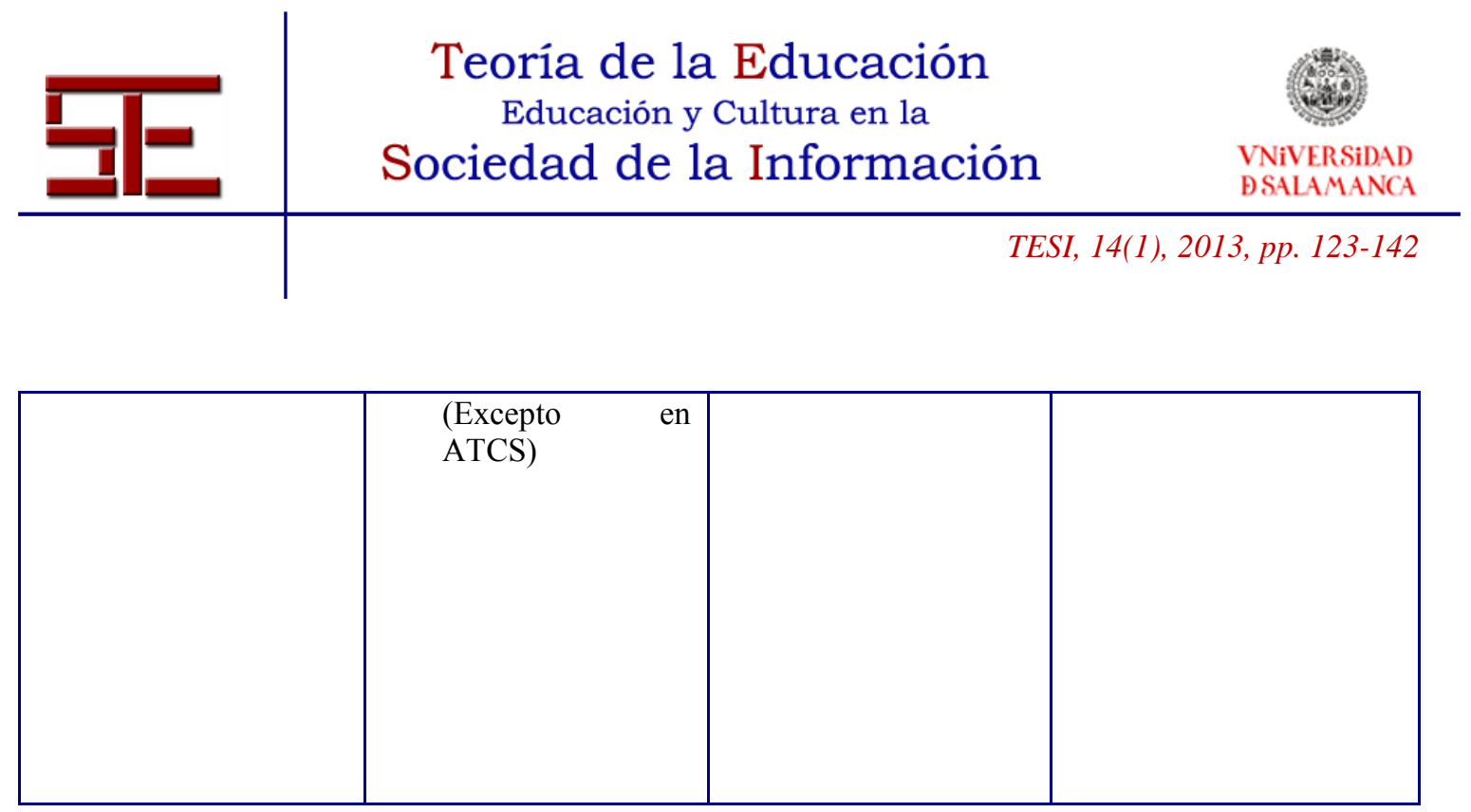

Como podemos observar en la Tabla 1 , las competencias de colaboración y comunicación aparecen como competencias clave mencionadas en todos los marcos de competencias del siglo XXI. Por ello, debemos considerar la importancia que tienen las competencias de colaboración para el futuro de la educación de ciudadanos del siglo XXI.

\section{2.- OPORTUNIDADES DE LOS SERIOUS GAMES PARA EL DESARROLLO DE LAS COMPETENCIAS DE COLABORACIÓN}

Las competencias de colaboración son esenciales en la sociedad actual, y deben ser también consideradas como un objetivo importante en la educación superior, tanto en los contextos de aprendizaje de carácter presencial, como semipresencial o virtual (Palloff y Pratt, 1999; Guitert, Giménez, Daradoumis y Marquès, 2000; Guitert, Guerrero, Romeu y Padrós, 2008). Estas competencias son necesarias también fuera del contexto educativo, en situaciones de educación no formal. En el ámbito profesional, cada vez más dinámico, es necesario desarrollar competencias que permitan la adaptación profesional, personal y social, que demandan las nuevas maneras de relacionarse (Monereo y Pozo, 2007).

Los juegos educativos digitales, también conocidos como Serious Games (SG), son un tipo de juegos destinados a finalidades educativas que apoyan e incentivan el aprendizaje centrado en el estudiante. Los educadores "utilizan juegos digitales con objetivos serios (es decir, con objetivos educativos) como herramientas para apoyar los procesos de aprendizaje de una manera significativa" (Sica, Delli Veneri y Miglino, 2011,108). Michael y Chen (2006) definen los SG como "un juego educativo en el que la educación (en sus diversas formas) es el objetivo principal, en lugar de entretenimiento" (p.17). Los SG tienen un potencial considerable en el desarrollo de las competencias de colaboración

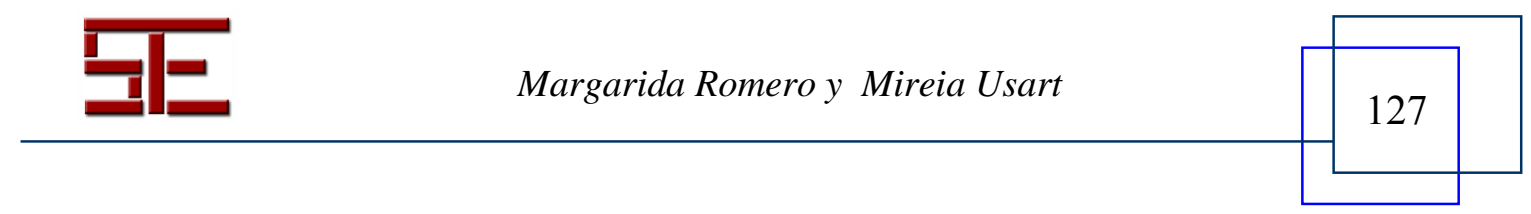




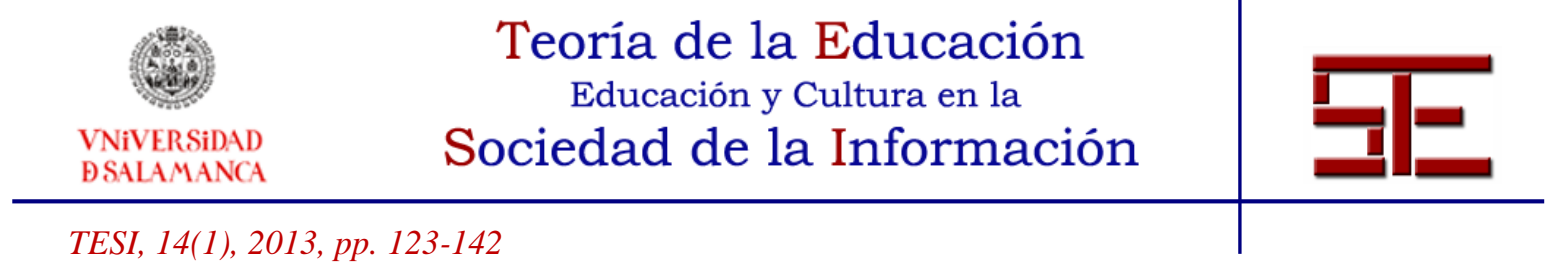

y la competición (Romero et al, 2012). El hecho de que un SG pueda ser considerado como una forma de aprendizaje activo, ya que sitúa al estudiante en el centro de la acción, y le exige un papel activo en el desarrollo de la actividad de aprendizaje basada en el juego (Oblinger, 2004). Además, la adopción de este Aprendizaje Basado en Juegos (ABJ) podría ayudar a los estudiantes en el desarrollo de las competencias de autorregulación (Bonwell, y Eison, 1991). También les permiten desarrollar las competencias digitales necesarias para la sociedad de la información (Imaz, 2011). El uso de juegos en contextos educativos puede ayudar a los estudiantes a alcanzar un cierto nivel de control en su actividad durante el juego educativo y así contribuir a un aprendizaje activo. Concretamente, las metodologías de ABJ se fundamentan en el aprendizaje activo y, a su vez, promueven actividades de aprendizaje mediante la construcción de conocimientos (Padrós, Romero y Usart, 2012), desarrolladas por los estudiantes cuando se implican en el juego educativo para lograr alcanzar los objetivos de aprendizaje previstos. Los SG ofrecen también un entorno seguro para que los estudiantes puedan explorar y experimentar sin los riesgos del mundo real (Garris, Ahlers y Driskell, 2002), y pueden contribuir a promover la colaboración creativa (Romero, Hyvönen y Barbera, 2012). Podemos observar, pues, que los juegos educativos digitales o SG pueden ser utilizados para incentivar el desarrollo de nuevas competencias para el siglo XXI (Prensky, 2006; Redecker et al, 2011).

Con el fin de analizar el impacto de los SG en el desarrollo de las competencias de colaboración entre los estudiantes adultos de educación superior, hemos desarrollado un estudio de caso con un SG concreto. Para ello, hemos seleccionado un contexto de educación formal para adultos e implementado uno de los SG desarrollados en el contexto del proyecto europeo GaLA NoE ${ }^{1}$ : el juego educativo MetaVals, que describiremos en las próximas secciones. Antes de analizar el caso de estudio de MetaVals analizamos en la siguiente sección las características de los juegos que permiten desarrollar las diferentes competencias del siglo XXI, centrándonos en las competencias colaborativas. En el análisis del potencial de los SG para el desarrollo de las competencias realizaremos una breve introducción de las razones que permiten apuntar sobre el potencial de los SG para el desarrollo de tales competencias. Después, describiremos en detalle el SG MetaVals, y

1 Game and Learning Alliance es una red de excelencia del Séptimo Programa Marco (7PM) de investigación y desarrollo de la Comunidad Europea, 2010-2014. http://www.galanoe.eu/.

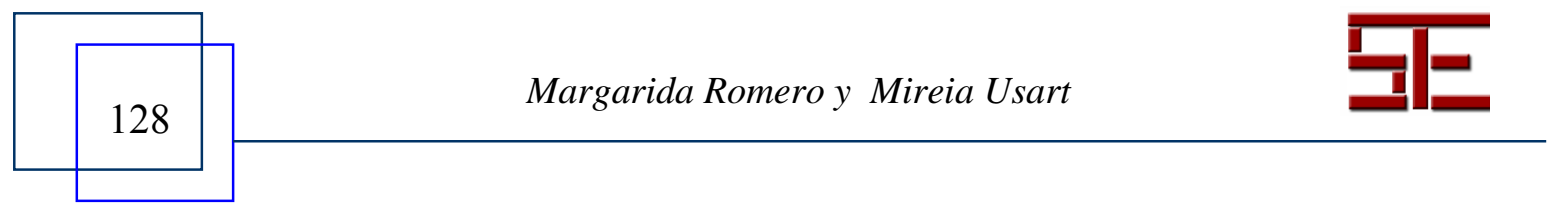




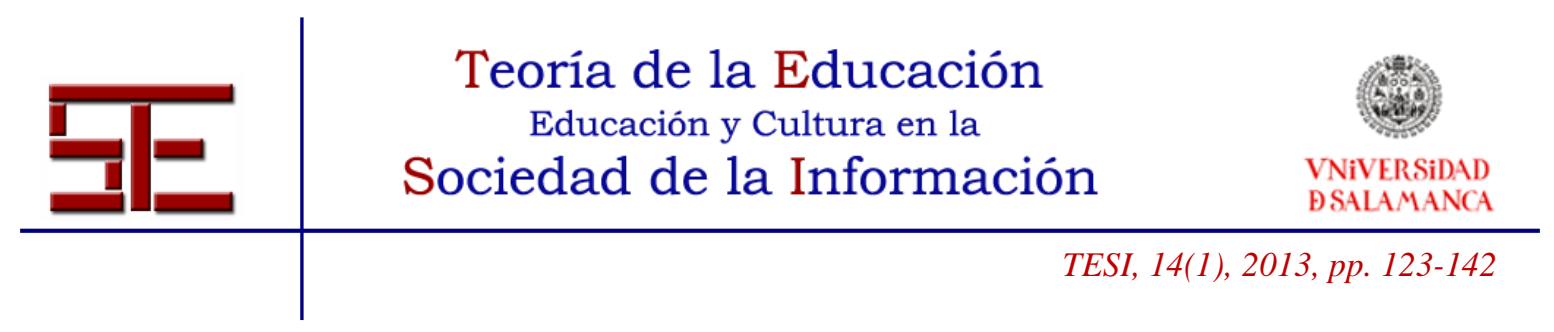

finalmente lo analizaremos en el marco de las características de los SG, para analizar cómo puede ayudar al desarrollo de las competencias de colaboración.

\section{3.- EL USO Y CARACTERÍSTICAS DE LOS SG EN EL DESARROLLO DE LAS COMPETENCIAS DE COLABORACIÓN}

Con el fin de analizar qué rol pueden jugar los SG en el desarrollo de las competencias de colaboración, y tras el análisis de la implementación de esta metodología ABJ en los contextos educativos en general, esta sección tiene como objetivo centrar el estudio de las diferentes características de los SG en relación con su potencial para el desarrollo de las competencias de colaboración. Las características de los juegos en general han sido estudiadas e identificadas por diferentes investigadores desde principios de los años 1950. Wittgenstein (1953) fue uno de los primeros autores en caracterizar el término "juego", incluyendo elementos tales como la partida, las reglas y la dinámica de competición en los juegos. Más tarde, Malone (1981) presentó como características propias de los juegos el desafío, la fantasía, la complejidad y el control. Según este autor, los atributos mencionados podrían ser utilizados para identificar el nivel de compromiso que incentiva cada juego en los jugadores. También Crookall, Oxford y Saunders (1987) identificaron dos características de juego diferentes: las reglas y las estrategias. En la misma línea, Thomas y Macredie (1994) indican que la característica principal de los juegos podría ser que las acciones llevadas a cabo dentro de éste no tienen consecuencias reales; esto podría permitir a los estudiantes practicar en un entorno seguro, sin temor al fracaso. Todas estas características de los SG podrían ayudar a los jugadores a adquirir conocimientos y ponerlos en práctica durante el transcurso del juego (Charsky, 2010).

En relación al potencial de los SG para ayudar a desarrollar y practicar las competencias de colaboración, podemos afirmar que las competencias sociales adecuadas para los sujetos que trabajarán con nuevas tecnologías son las interpersonales y las colaborativas (Alfageme y Sánchez, 2002). Según los autores, éstas se pueden tratar de potenciar con el uso de los juegos digitales en la educación formal, ya que el aprendizaje que se obtiene de una situación concreta se puede utilizar posteriormente para resolver problemas, mediante la experiencia necesaria para la formación de su personalidad y conducta social hábil. En la Tabla 2 se presenta la taxonomía de características de los juegos basada en las obras de Garris, Ahlers y Driskell (2002) y de Charsky (2010). Esta clasificación nos permitirá identificar qué características en concreto podrían apoyar el desarrollo de competencias de colaboración. La taxonomía presentada prevé cinco características principales (columna de la izquierda/elementos en negrita). Siguiendo el trabajo de Charsky, también se ha indicado un segundo nivel de clasificación (columna de la

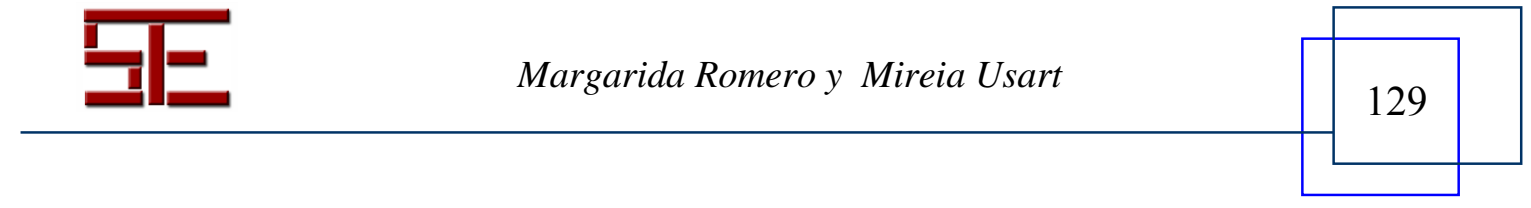




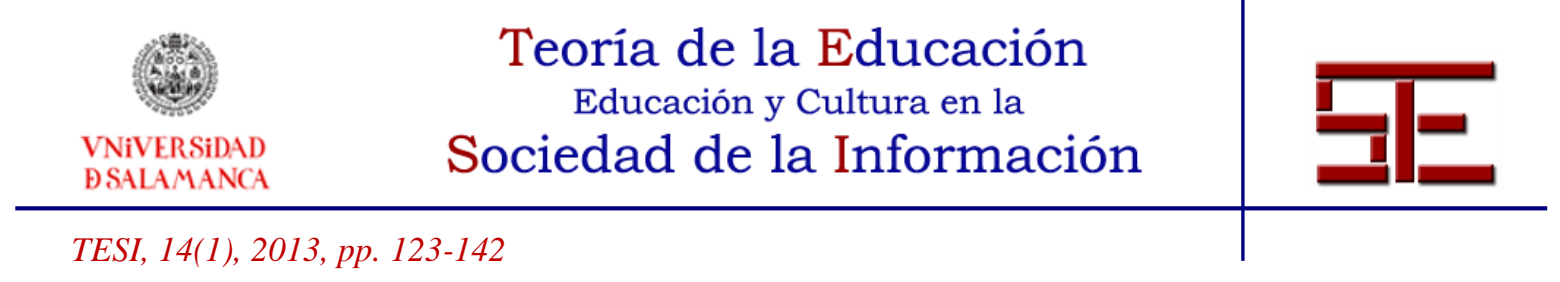

derecha), para realizar una clasificación más completa de cada característica, útil para nuestros propósitos. Finalmente, en la tercera columna, cada característica de juego es analizada en términos de interés para el desarrollo de las diferentes competencias.

Tabla 2. Taxonomía de las características de los juegos (Garris, Ahlers \& Driskell, 2002; Charsky, 2010)

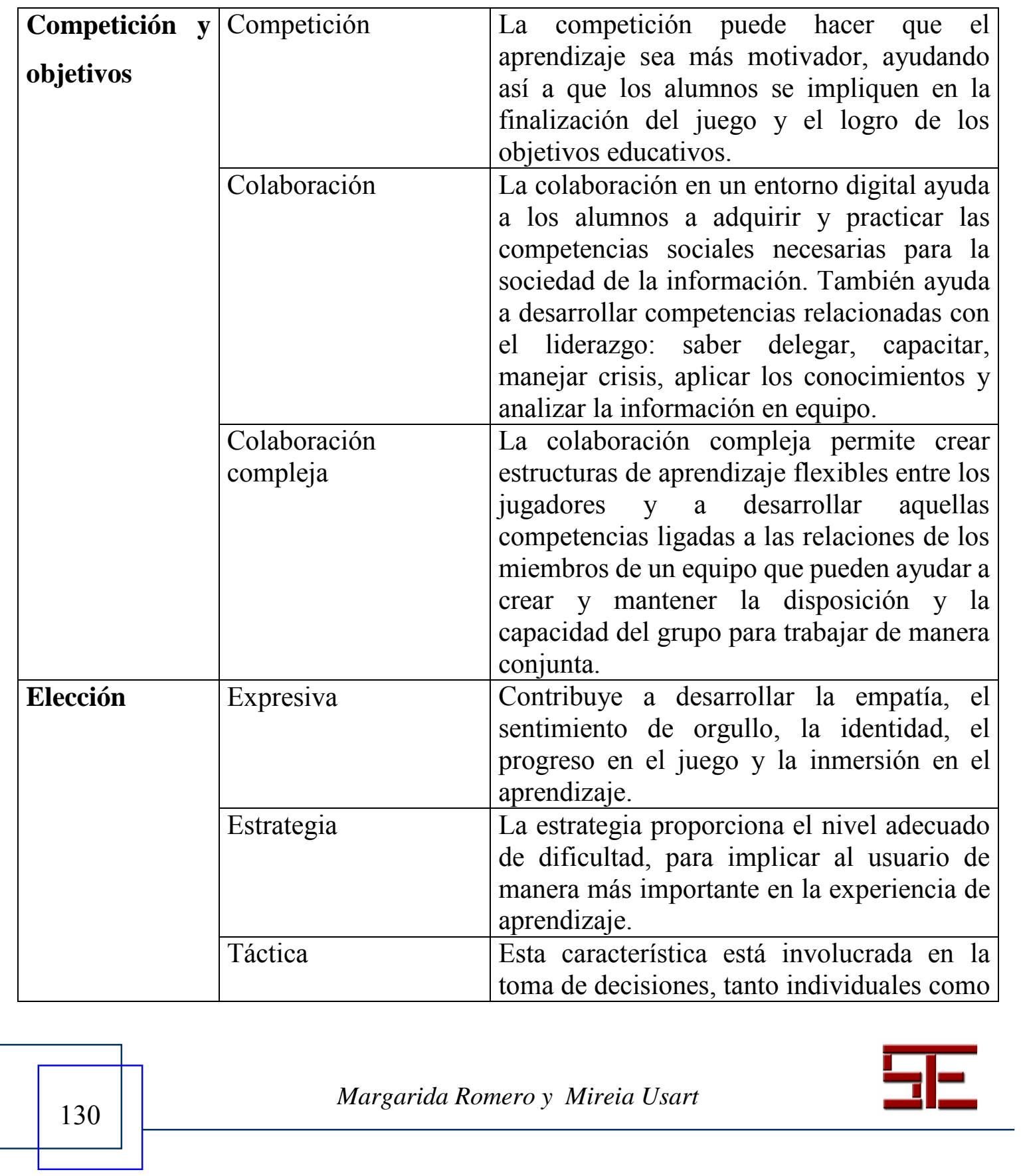




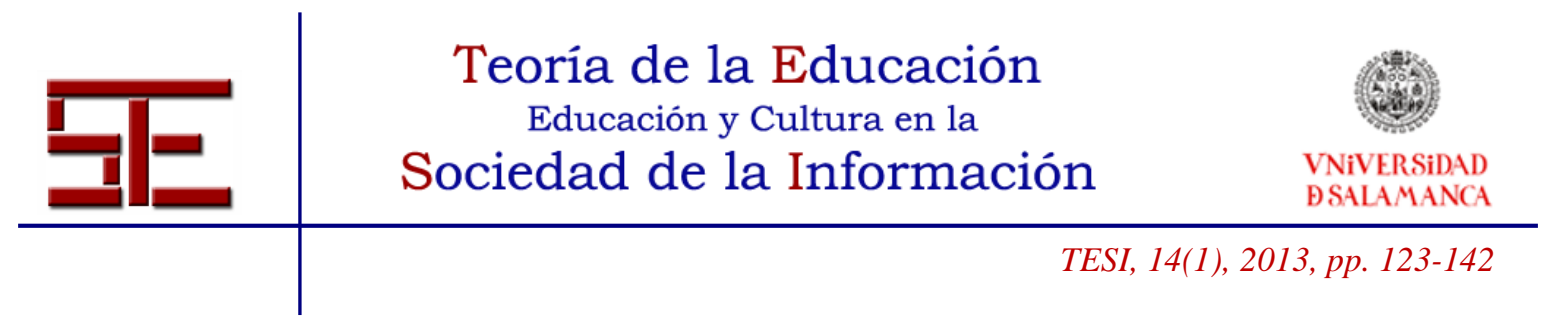

\begin{tabular}{|l|l|l|} 
& & $\begin{array}{l}\text { colaborativas, así como en la adaptación de } \\
\text { las competencias reales para apoyar el } \\
\text { aprendizaje ofreciendo información clave, } \\
\text { asistencia, asesoramiento u orientación. }\end{array}$ \\
\hline Reglas & $\begin{array}{l}\text { Las reglas permiten desarrollar } \\
\text { competencias tanto de orden inferior como } \\
\text { de orden superior, ya que introducen } \\
\text { limitaciones que se deben resolver y } \\
\text { demandan del jugador poner en práctica sus } \\
\text { conocimientos previos. }\end{array}$ \\
\hline Fantasía & Fidelidad & $\begin{array}{l}\text { La fidelidad del juego respecto al contexto } \\
\text { de la realidad representado contribuye a la } \\
\text { inmersión de cada participante en el juego } \\
\text { concreto, mediante la recreación más o } \\
\text { menos realista del escenario que se pretende } \\
\text { representar. }\end{array}$ \\
\hline Contexto & $\begin{array}{l}\text { El contexto permite al estudiante tener una } \\
\text { experiencia de aprendizaje más auténtica, } \\
\text { cercana al mundo real en el que deberá } \\
\text { posteriormente aplicar los conocimientos } \\
\text { adquiridos. }\end{array}$ \\
\hline Reto & $\begin{array}{l}\text { El reto que caracteriza todos los juegos } \\
\text { podría ayudar en la reflexión y procesos } \\
\text { metacognitivos de los jugadores, aportando } \\
\text { un nivel optimo de dificultad e } \\
\text { incertidumbre, el denominado flow } \\
\text { (Csikszentmihalyi, 1997), que permita } \\
\text { trabajar sobre los objetivos a conseguir de } \\
\text { manera activa y consciente. }\end{array}$ \\
\hline
\end{tabular}

\section{4.- ANALISIS DEL ESTUDIO DE CASO: METAVALS}

Hemos explorado la cuestión de si los SG pueden ayudar a desarrollar las competencias colaborativas, y cómo pueden hacerlo mediante las características que definen a los

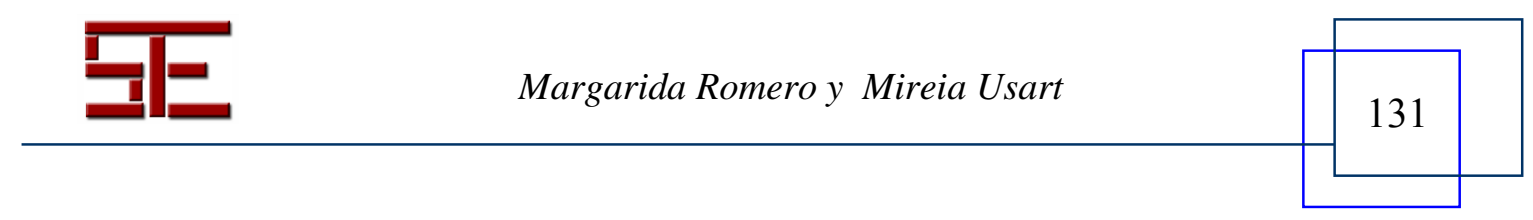




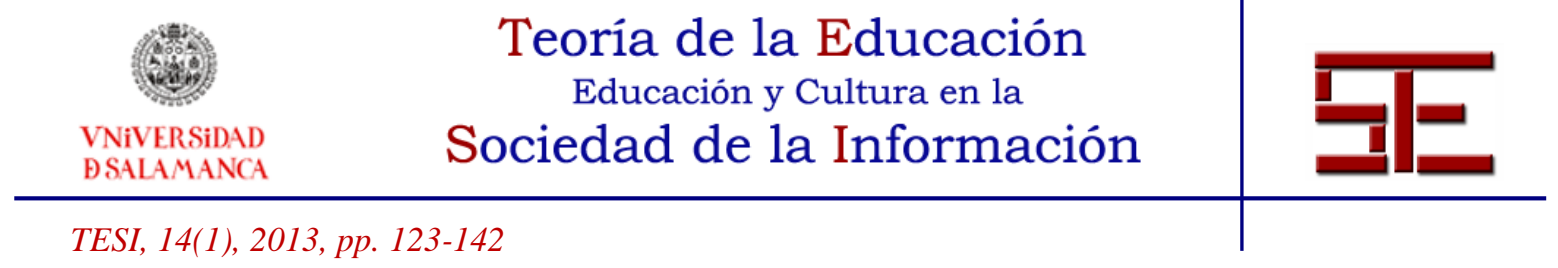

juegos digitales. Gracias a un minucioso examen de la literatura hemos visto cómo los SG ya se han utilizado con anterioridad en diferentes entornos de aprendizaje para propiciar, de manera efectiva, el desarrollo de gran parte de las competencias. A partir de aquí podemos deducir que los SG, efectivamente, pueden ser considerados como una herramienta adecuada para dar soporte y promover el desarrollo de las competencias, concretamente, las competencias de colaboración. En cuanto a cómo los SG pueden contribuir a solucionar este problema, hemos mostrado que las principales características de los juegos cubren la mayoría de las competencias de colaboración identificadas y hemos podido emparejar cada una de las competencias con una característica específica.

A modo de ejemplo práctico, reportamos en esta sección el estudio de caso centrado en el juego educativo Metavals. Mostramos a continuación cómo un SG diseñado específicamente para su aplicación en entornos educativos puede por sí solo ayudar en la práctica de nuevas competencias, concretamente, la práctica de las competencias de colaboración. En primer lugar, vamos a describir el juego MetaVals con la finalidad de establecer el escenario de estudio concreto. En segundo lugar, presentaremos cada una de las características de los SG enumeradas en el apartado anterior para el ejemplo concreto de MetaVals. Este paso es clave para asegurar que el diseño de este SG está en línea con la taxonomía presentada anteriormente. Finalmente, cada una de las competencias de colaboración identificadas también se relacionará con el SG MetaVals, con el fin de presentar qué competencias concretas se pueden practicar a través de la utilización de este SG.

\section{1.- Descripción del juego MetaVals}

MetaVals es un juego educativo digital, diseñado por el Departamento de Innovación pedagógica y calidad académica (DIPQA) de la escuela de negocios ESADE en el contexto de la red de excelencia del Séptimo programa marco (7PM) de investigación y desarrollo de la Comunidad Europea llamado Games and Learning Alliance (GALA). Este SG fue adaptado a partir de una actividad existente que se realizaba en clase de manera oral mediante la interacción entre el profesor y los alumnos. Esta actividad de aprendizaje en el aula fue diseñada para la práctica de los conceptos financieros básicos: los activos y los pasivos (Massons et al., 2011). A pesar del interés pedagógico de la actividad inicial, y debido a la naturaleza de la misma, sólo unos pocos estudiantes mostraban un papel activo durante el desarrollo de la actividad en el aula. En este contexto era difícil incentivar la discusión entre alumnos o entre alumnos y profesor. Sobre la base de esta actividad presencial, el juego MetaVals fue diseñado para vencer estas dificultades, mediante un proceso que se inició con una primera versión en papel del

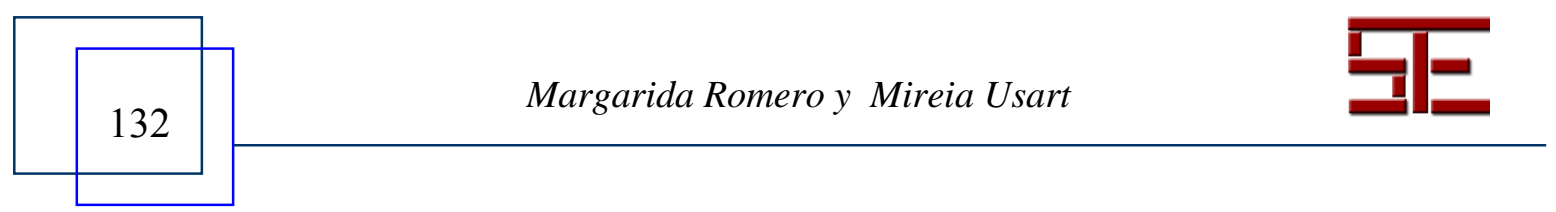




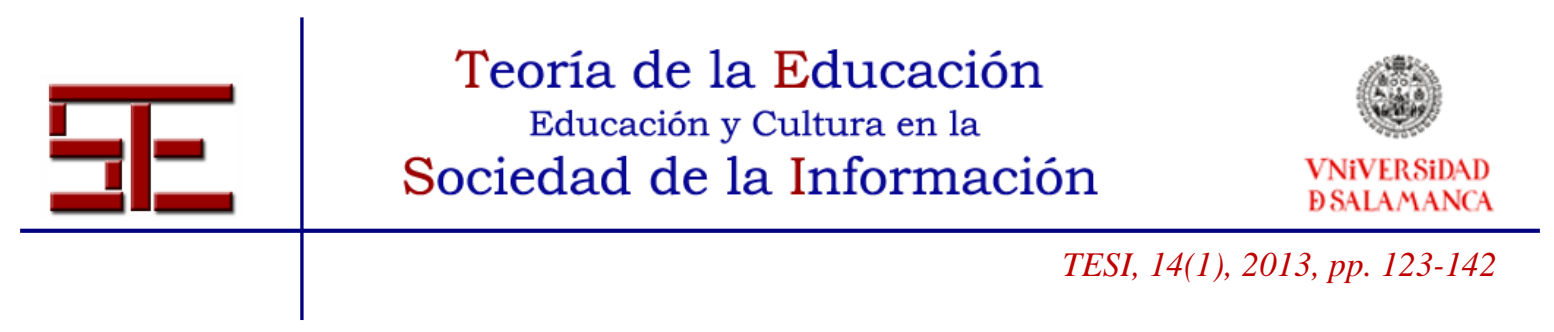

juego, totalmente presencial, a la que siguieron dos versiones digitales las cuales se pusieron a prueba en entornos reales de aprendizaje, con diferentes grupos de estudiantes adultos de postgrado (Padrós, Romero y Usart, 2011). En su versión actual, el SG MetaVals se puede definir como un juego de clasificación en tres fases, donde los estudiantes juegan por parejas (o díadas) contra el resto del grupo-clase. Actualmente se puede implementar el juego para que los estudiantes tengan un compañero real o bien uno virtual, dependiendo del contexto y situación de aprendizaje en el que se implementa el juego. Al inicio del juego aparece una pantalla de bienvenida que invita a los jugadores a introducir su edad, nivel de conocimientos previos y experiencia previa en finanzas (en una escala de 0 a 10). Acto seguido se invita a los jugadores a rellenar una prueba de conocimiento previo diseñada por profesores expertos en la docencia de los contenidos tratados en el juego (finanzas, estadística) con la finalidad de comparar el conocimiento previo autorreportado con el resultado de este test previo. La segunda pantalla lleva a los jugadores a un escenario diferente que contiene la información de sus compañeros (virtuales o reales; ver Figura 1). Los datos que se muestran pretenden ayudar a cada jugador durante las dos fases colaborativas del juego (corrección y debate). Por ejemplo, un compañero con un bajo nivel en finanzas puede dar respuestas incorrectas, e indicar así a su pareja que tal vez necesita más ayuda durante la fase siguiente.

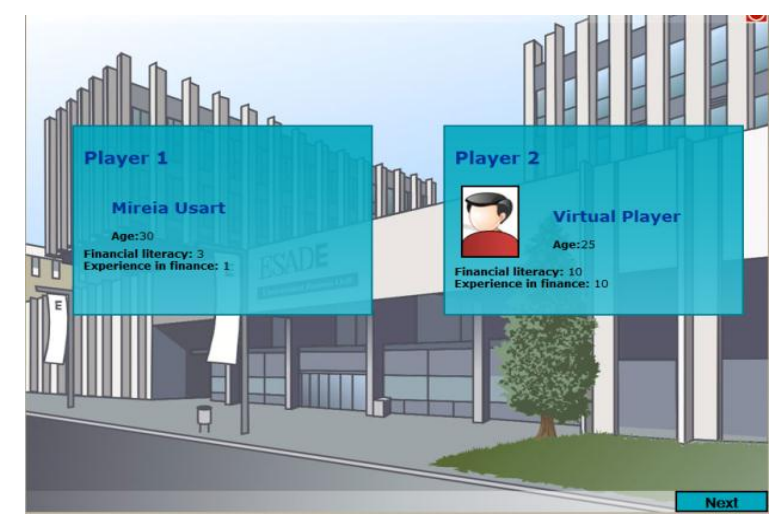

Figura 1. Pantalla del juego MetaVals en el que el jugador ve la información de su compañero.

Después de las instrucciones generales y la explicación de las reglas del juego, presentadas por un avatar virtual del profesor, el jugador puede empezar a jugar la fase individual. En primer lugar, el jugador debe clasificar por separado seis ítems (ej. como activos o pasivos: "Aplicaciones informáticas", "Crédito bancario"); para cada ítem, el

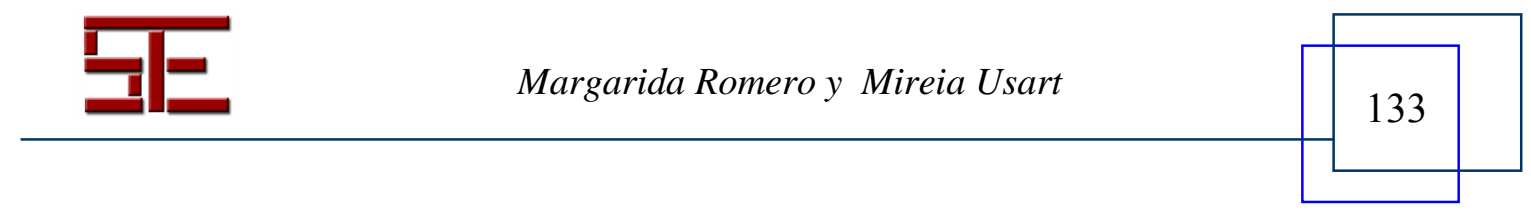




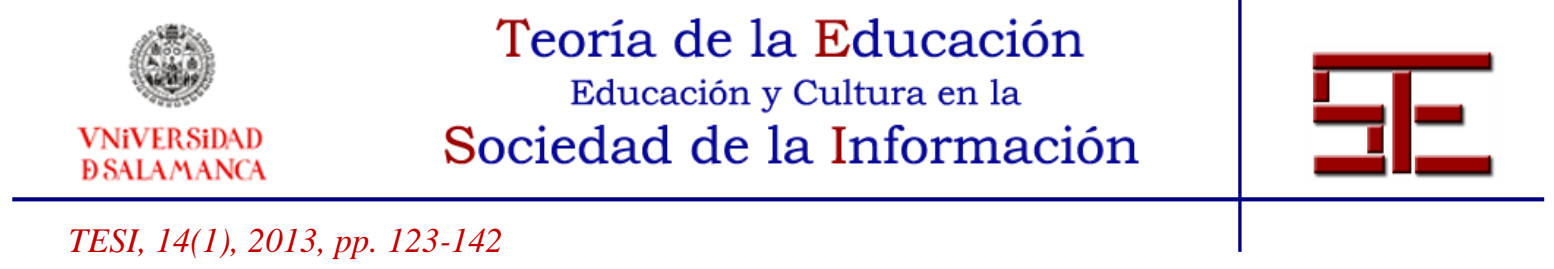

jugador puede indicar su nivel de certidumbre en una escala del 0 al 10 (si está completamente seguro, que puede anotar un 10, si no está nada seguro, un 0), ver Figura 2. Después de esta primera fase, otros seis ítems diferentes aparecen, pero ahora el jugador tiene acceso a las respuestas y nivel de certidumbre que su compañero de juego acaba de indicar. Después de esta fase de corrección, empieza la tercera fase, la fase de discusión. En esta última fase, los dos jugadores juntos tienen que decidir si los 12 ítems previamente clasificados son correctos, y dar así una respuesta final consensuada. La díada con el mayor número de respuestas correctas en el menor tiempo gana el juego (cada pantalla dispone de un reloj de cuenta atrás que permite a los jugadores saber cuánto tiempo de juego llevan, con un máximo de 10 minutos por fase). Finalmente, aparece la pantalla de clasificación, donde los estudiantes pueden visualizar la posición final de su díada dentro del conjunto del grupo-clase y acceder a un cuestionario final de valoración de la experiencia de juego (post-test).

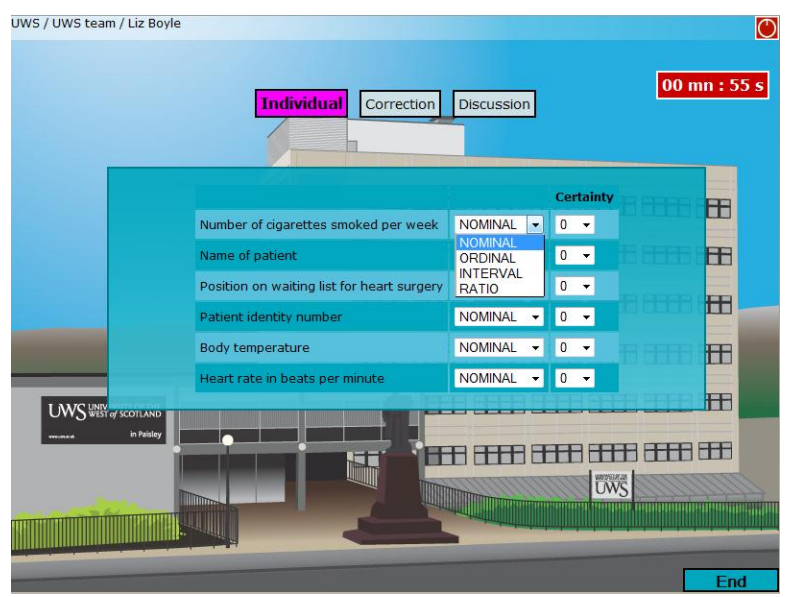

Figura 2. Pantalla de la fase individual del SG MetaVals.

La versión actual del juego MetaVals es altamente personalizable y se puede adaptar a diferentes campos de conocimiento, tanto a nivel gráfico como de contenidos. De hecho, la versión actual del juego ha sido probada en diferentes cursos de finanzas, en dos clases de estadística y en una clase de terminología financiera en inglés en diferentes países y siempre con estudiantes adultos (Romero, Usart, Popescu y Boyle, 2012). Esta versión actual implementa un reloj de cuenta atrás para cada una de las fases del juego, que se muestra en la pantalla y se registra en una base de datos MySQL, con el fin de controlar las puntuaciones de los estudiantes y el tiempo invertido en el juego, tanto para la fase

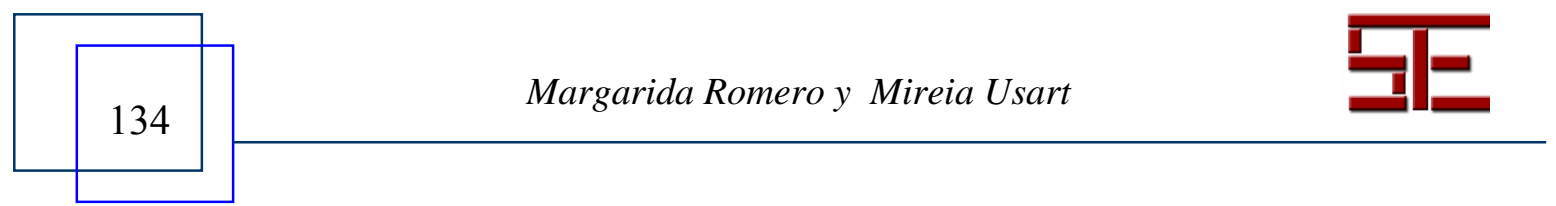




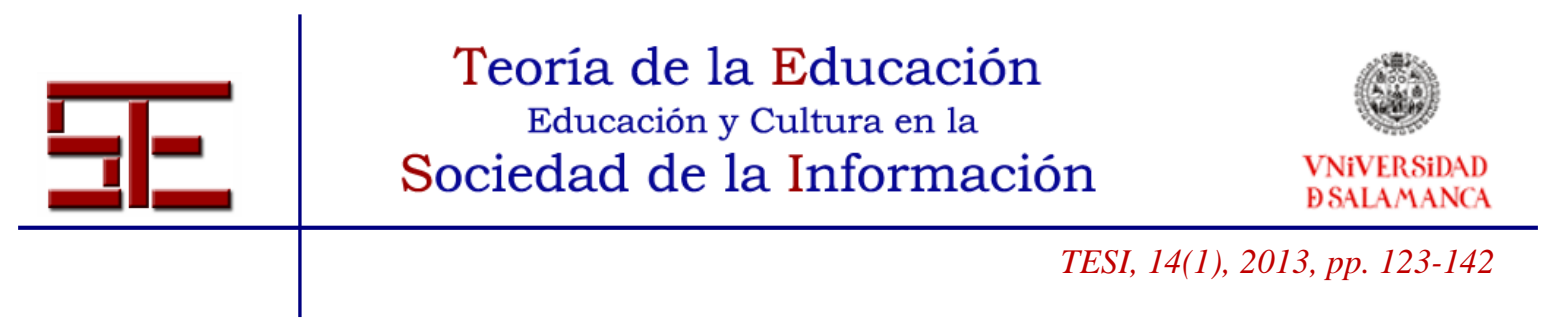

individual como para las dos fases colaborativas. El SG también permite monitorizar el nivel previo de conocimientos y experiencia en el campo concreto de conocimiento, junto con la edad, el género y el avatar elegido por cada participante. Todos estos datos pueden dar a los investigadores y profesores una visión completa de cada partida y así estudiar el proceso de aprendizaje durante el juego.

\subsection{Características del SG MetaVals}

Una vez presentado con detalle el SG MetaVals, procedemos a analizar en detalle las competencias que se pretenden practicar mediante esta actividad. En la Tabla 3 podemos observar las características del juego educativo MetaVals; esta tabla se ha realizado de acuerdo con la clasificación introducida por Charsky (2010), explicada en los apartados anteriores.

Tabla 3. Análisis de las características del SG MetaVals

\begin{tabular}{|c|c|c|}
\hline \begin{tabular}{|l} 
Categoría de la \\
característica
\end{tabular} & Característica & MetaVals \\
\hline \multirow[t]{3}{*}{$\begin{array}{l}\text { Competición } \\
\text { objetivos }\end{array}$} & Competición & $\begin{array}{l}\text { MetaVals permite la competición a } \\
\text { nivel intergrupal (nivel de grupo- } \\
\text { clase). Las díadas pueden comparar } \\
\text { sus puntuaciones al final del juego y } \\
\text { ver el ranking final de la partida. }\end{array}$ \\
\hline & Colaboración & $\begin{array}{l}\text { El juego por díadas permite promover } \\
\text { la colaboración intragrupal; esto se } \\
\text { consigue creando interdependencia } \\
\text { durante la última fase del juego, } \\
\text { donde los estudiantes deben decidir } \\
\text { de manera conjunta (díada) la } \\
\text { respuesta final para cada ítem. }\end{array}$ \\
\hline & $\begin{array}{l}\text { Colaboración } \\
\text { compleja }\end{array}$ & $\begin{array}{l}\text { La colaboración está estructurada } \\
\text { durante todo el SG (guiada a través de } \\
\text { las diferentes pantallas). MetaVals no } \\
\text { proporciona un apoyo concreto para } \\
\text { la colaboración compleja, ya que se } \\
\text { basa en una estructura fuertemente } \\
\text { guiada que permita a los estudiantes }\end{array}$ \\
\hline
\end{tabular}

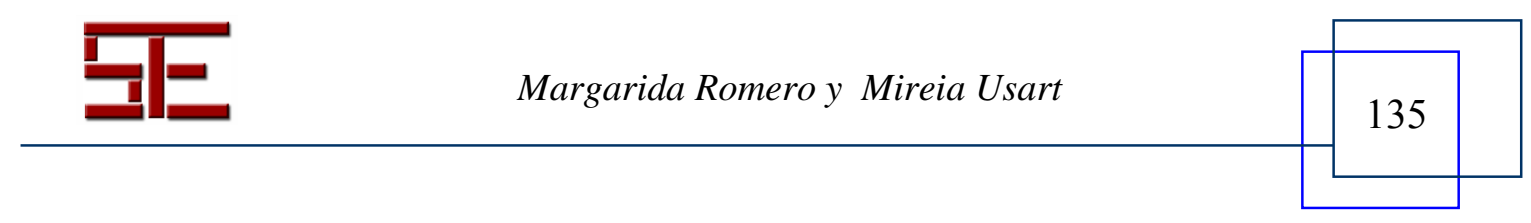




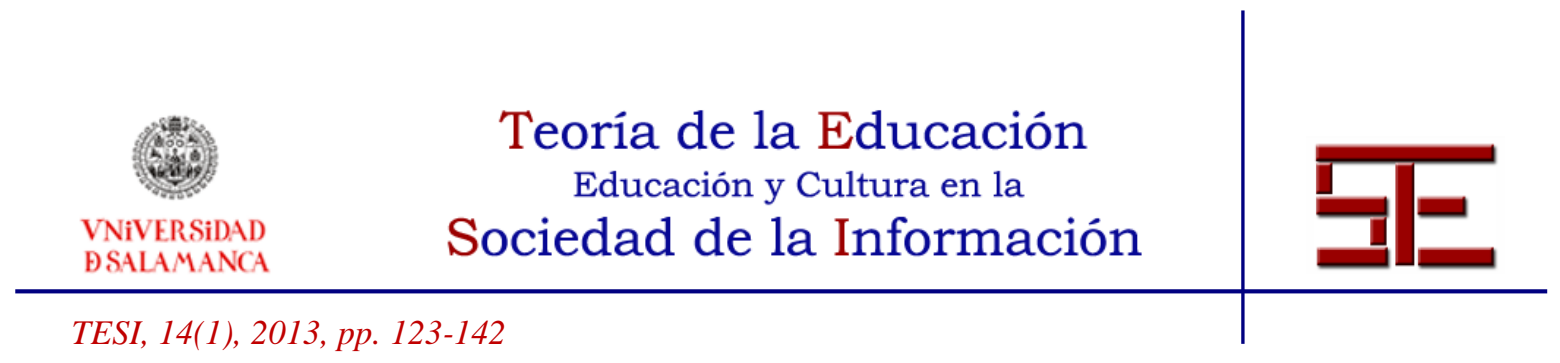

\begin{tabular}{|c|c|c|}
\hline & & $\begin{array}{l}\text { centrarse en la discusión por parejas } \\
\text { de los ítems propuestos. }\end{array}$ \\
\hline \multirow[t]{3}{*}{ Elección } & Expresiva & $\begin{array}{l}\text { MetaVals permite a los estudiantes } \\
\text { personalizar sus avatares de manera } \\
\text { individual, declarar su edad y nivel de } \\
\text { conocimientos y experiencia previos } \\
\text { en el campo de conocimientos } \\
\text { considerado (ej. finanzas, estadística). }\end{array}$ \\
\hline & Estrategia & $\begin{array}{l}\text { MetaVals está altamente estructurado } \\
\text { y no promueve el desarrollo de } \\
\text { estrategias muy elaboradas por parte } \\
\text { de los participantes. }\end{array}$ \\
\hline & Táctica & $\begin{array}{l}\text { MetaVals no promueve de manera } \\
\text { explícita el desarrollo de tácticas, sin } \\
\text { embargo, cuando se juega en díadas, } \\
\text { los jugadores pueden decidir cómo } \\
\text { utilizar el nivel de certidumbre, } \\
\text { pueden elegir si compartir, mentir o } \\
\text { decir la verdad a su compañero en este } \\
\text { aspecto, para favorecer o dificultar los } \\
\text { procesos metacognitivos } \\
\text { compartidos. }\end{array}$ \\
\hline Reglas & \multicolumn{2}{|c|}{$\begin{array}{l}\text { El juego MetaVals presenta un personaje virtual (ej. profesor) } \\
\text { que explica las diferentes reglas de juego y las recuerda antes } \\
\text { de empezar las diferentes fases del juego. Esto permite que el } \\
\text { alumno comprenda las reglas de cada una de las fases, la fase } \\
\text { individual y las dos fases de colaboración. Estas reglas son } \\
\text { estrictas, y si los jugadores no las siguen de manera tanto } \\
\text { individual como colaborativa, no puede finalizar el juego. }\end{array}$} \\
\hline Fantasía & Fidelidad & $\begin{array}{l}\text { El juego MetaVals es un juego basado } \\
\text { en la web, y diseñado en 2D. Fue } \\
\text { ideado para que ofreciera una imagen } \\
\text { profesional que permitiera situar a los } \\
\text { estudiantes en un entorno en el que } \\
\text { tuvieran que tomar decisiones (ej. } \\
\text { Entorno financiero). Por otro lado, en } \\
\text { este juego no hay elementos }\end{array}$ \\
\hline
\end{tabular}

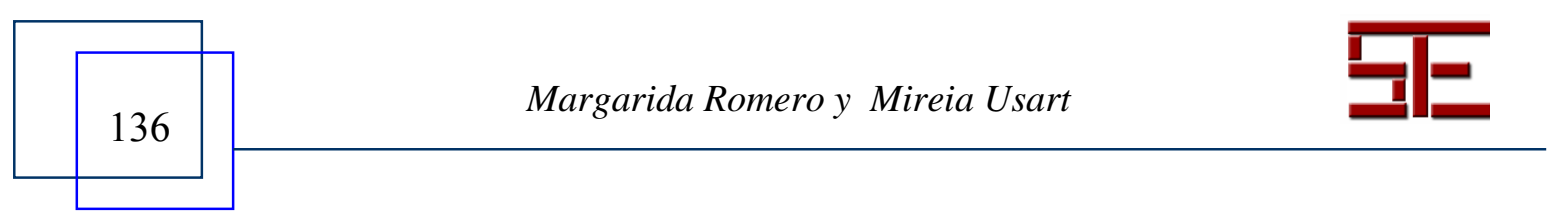




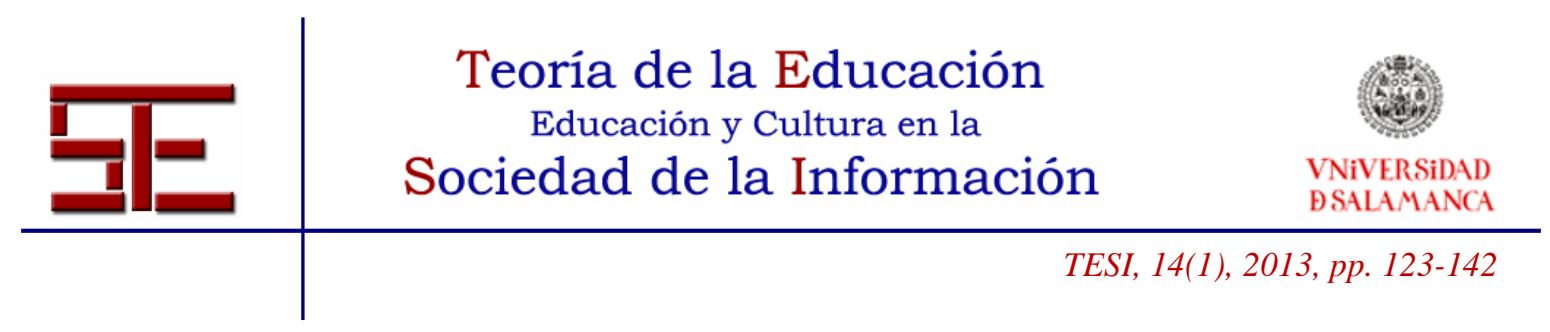

\begin{tabular}{|l|l|} 
& $\begin{array}{l}\text { fantásticos, podemos así afirmar que } \\
\text { MetaVals es un juego basado en la } \\
\text { realidad. }\end{array}$ \\
\cline { 2 - 3 } & Contexto \\
\hline Reto & $\begin{array}{l}\text { El objetivo de MetaVals es presentar y } \\
\text { desarrollar una historia con personajes } \\
\text { y situaciones que ayude a los } \\
\text { estudiantes no simplemente a seguir la } \\
\text { historia, si no a crear de manera activa } \\
\text { la narración a través de sus decisiones } \\
\text { y actuaciones durante el transcurso de } \\
\text { la partida. }\end{array}$ \\
\hline $\begin{array}{l}\text { Para ganar una partida en el juego MetaVals los jugadores } \\
\text { deben resolver un problema planteado en 3 fases. Cada } \\
\text { jugador puede mostrar a su compañero de díada si tiene un } \\
\text { conocimiento mayor en el área de conocimiento concreto (ej. } \\
\text { Finanzas). Este juego fue diseñado también mediante el } \\
\text { establecimiento de diferentes niveles de dificultad, los ítems } \\
\text { pueden ser de nivel de dificultad bajo, medio o alto, y en el } \\
\text { momento de la implementación del juego, estos combinan } \\
\text { con el fin de añadir un mayor grado de desafío al juego. }\end{array}$ \\
\hline
\end{tabular}

\subsection{Desarrollo de las competencias de colaboración a través de MetaVals}

El juego educativo analizado, MetaVals, tiene como objetivo principal incentivar y desarrollar la toma de decisiones colectivas a nivel de díadas de aprendizaje (Romero, Usart y Almirall, 2011). Así, el SG MetaVals permite desarrollar las competencias de colaboración mediante la interacción entre los dos jugadores que forman las díadas, creadas para cada partida. Esta colaboración se puede llevar a cabo con un jugador virtual o con una pareja real, dependiendo del escenario en el que se implemente MetaVals. En el caso de díadas reales, habitualmente contemplado para contextos de formación presenciales o semipresenciales, donde el SG MetaVals se juega en el aula utilizando ordenadores portátiles o tabletas, las díadas pueden interaccionar durante la tercera fase del juego mediante una herramienta de chat (ej. chat Moodle). De esta manera se pretende facilitar la discusión y toma de decisiones en la fase final, colaborativa, del juego. También es importante destacar el uso de la herramienta "nivel de certidumbre" en todas

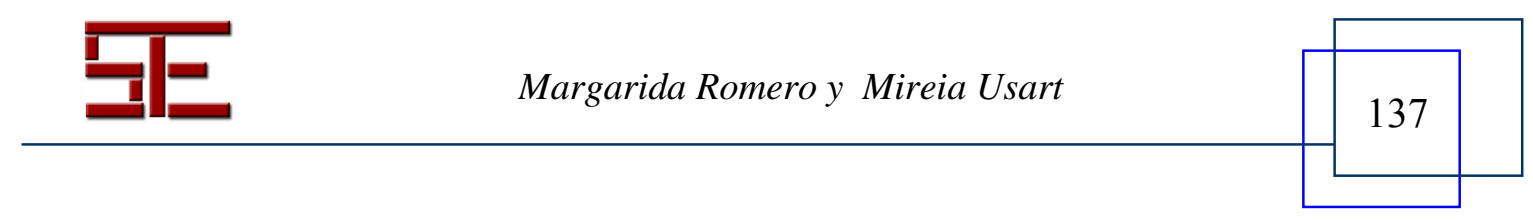




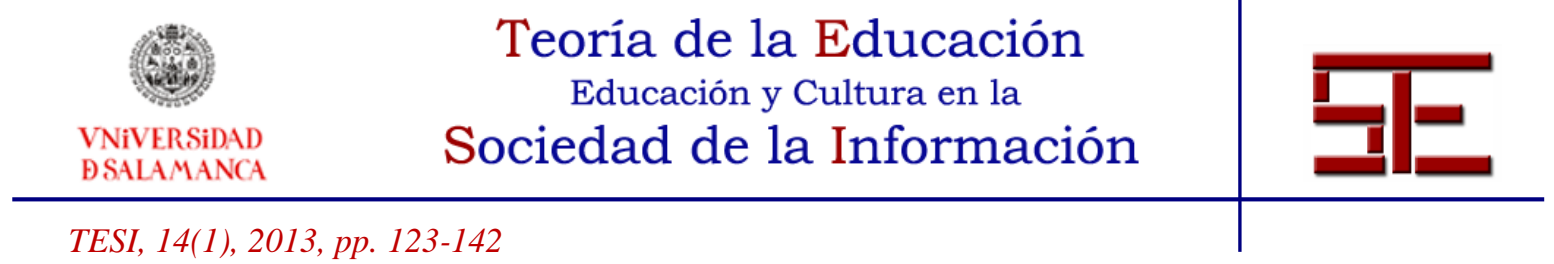

las fases del juego. Mediante el uso de esta herramienta se pretende ayudar a los jugadores en sus procesos metacognitivos de aprendizaje (Usart, Romero \& Almirall, 2011); el hecho de permitir que cada participante explicite su nivel de certidumbre y lo comparta con su compañero de juego puede ayudar a reflexionar de manera consciente sobre cada respuesta y llegar a un diálogo más focalizado en el contenido, que finalmente ayude a una mejor actuación en el juego.

\section{5.- DISCUSIÓN}

El objetivo principal de este estudio ha sido el de examinar si los juegos digitales, y concretamente los SG, pueden ayudar al desarrollo de las competencias de colaboración, y de qué modo lo pueden hacer. Después de un examen en profundidad de la literatura, tanto en el campo de los juegos y sus características como en el de las competencias de aprendizaje, nos hemos centrado en un juego educativo concreto; el SG MetaVals, para estudiar en detalle cómo un juego educativo digital diseñado a propósito puede ayudar a practicar las competencias colaborativas.

El hecho de que MetaVals haya sido diseñado e implementado mediante un proceso iterativo, a partir de una actividad en el aula, por un equipo de diseñadores instruccionales junto con un profesor experto podría ser un factor clave que permita el desarrollo completo de las diferentes características de juego, y la adecuación de cada una de estas características a los objetivos educativos, concretamente, al desarrollo de las competencias colaborativas de los estudiantes. Así, las características estudiadas, concretamente las de colaboración y competición, se podrían utilizar como herramientas de los SG para el desarrollo de diferentes competencias de colaboración, en concreto, para las competencias sociales o de relación (Alfageme \& Sánchez, 2002). Como ejemplo concreto, el uso de gráficos de calidad en 2D, adaptados a cada contenido, y la implementación de personajes o avatares durante el juego, así como las características de fidelidad y reto, deberían ayudar al desarrollo, en los estudiantes, de diferentes competencias tales como el pensamiento crítico y la resolución de problemas a través el juego.

Durante este estudio hemos visto que, aparte de los objetivos de contenido, es importante tener en cuenta las competencias concretas que se quieren desarrollar mediante el uso de SG, y aplicar así las características adecuadas para conseguirlo. En concreto, y dentro del contexto de las competencias del siglo XXI, tanto la colaboración cómo la competición eran dos aspectos fundamentales que perseguían los diseñadores en el momento de idear

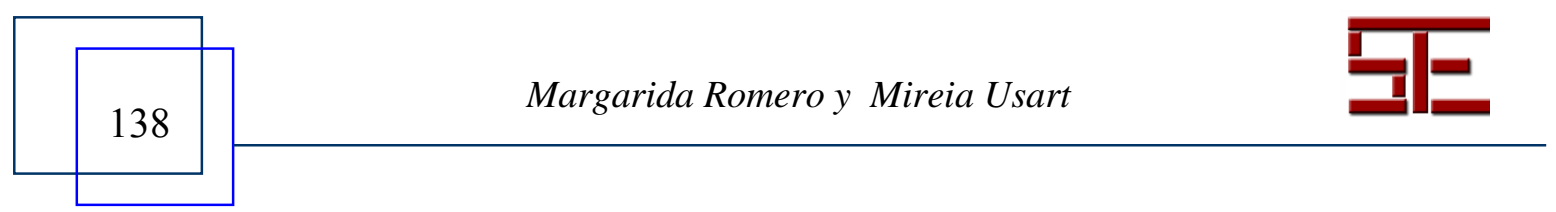




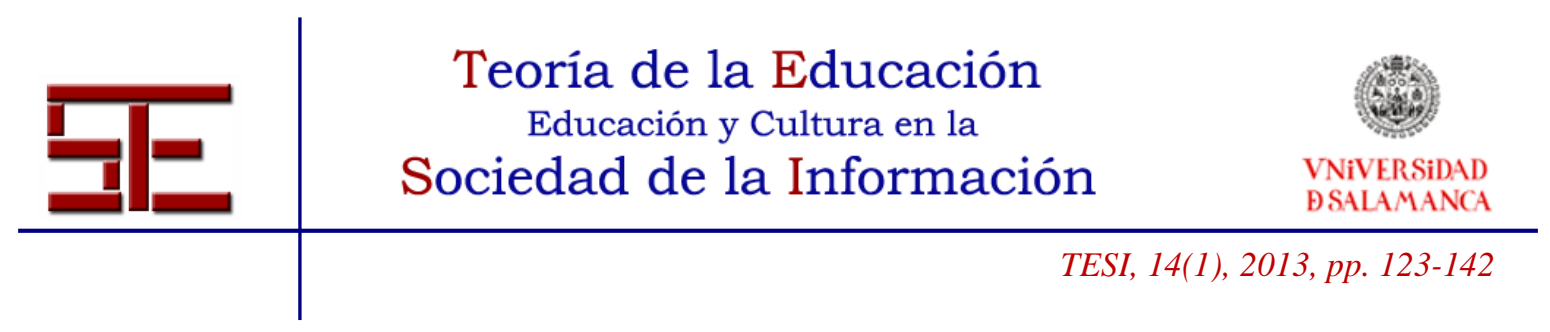

el juego educativo MetaVals, ya que estas dos competencias pueden ser consideradas como centrales tanto en los estudios los negocios y gestión (Mawdesley, Largo, AlJibouri y Scott, 2011) como en los contextos evaluativos en el ámbito universitario (Olmos-Migueláñez y Rodríguez-Conde, 2011).

\section{6.- CONCLUSIONES}

El desarrollo de competencias del siglo XXI, y concretamente las competencias de colaboración, aún no se ha explorado de manera extensa en el contexto educativo formal, sin embargo, hemos visto cómo los juegos digitales educativos o SG implementados en contextos de aprendizaje activo podrían ser herramientas que ayuden a los estudiantes a adquirir y practicar estas competencias, como se deriva del estudio de caso del SG MetaVals y de cada una de las características del juego. No obstante, existe la necesidad de diseñar los SG desde un punto de vista educativo, que tenga en cuenta los objetivos, no sólo de contenido, sino también competenciales, de cada acción formativa. A partir de este principio, se deben explicitar las competencias que se quieran practicar durante el juego, y establecer de qué manera las características de los SG nos pueden ayudar. No se trata de cubrir una amplia gama de competencias en cada juego, sino de crear juegos de corta duración que se puedan implementar en el currículo educativo como actividades centradas en el estudiante y en la práctica competencial, y que sean diseñados específicamente para entrenar un número limitado de competencias en un mismo juego.

Creemos pues que las investigaciones futuras en el campo deben tener en cuenta la inclusión de aquellas características de los juegos que directamente podrían desempeñar un papel en el desarrollo y la práctica de las diferentes competencias de colaboración, en combinación con los objetivos de aprendizaje.

\section{7.- BIBLIOGRAFÍA}

Alfageme, B. y Sánchez, P. (2002). Aprendiendo habilidades con videojuegos. Comunicar, 19, 114-119.

Barrio de la Puente, J.L. (2009). Hacia una educación inclusiva para todos. Teoría de la Educación, 20 (1), 13-31.

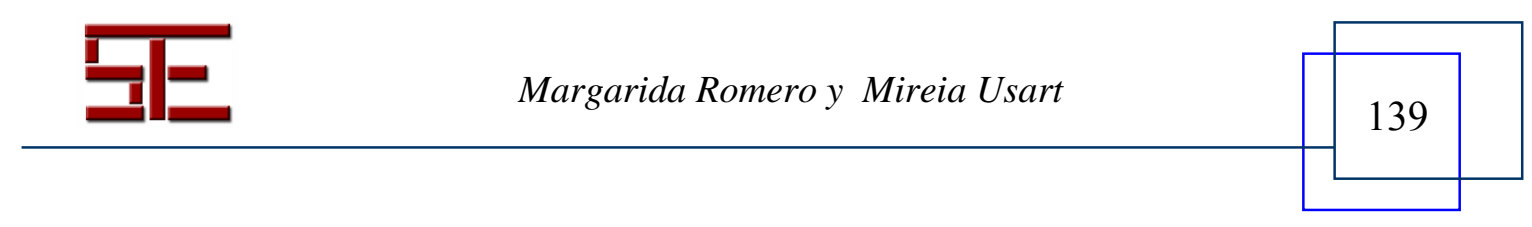




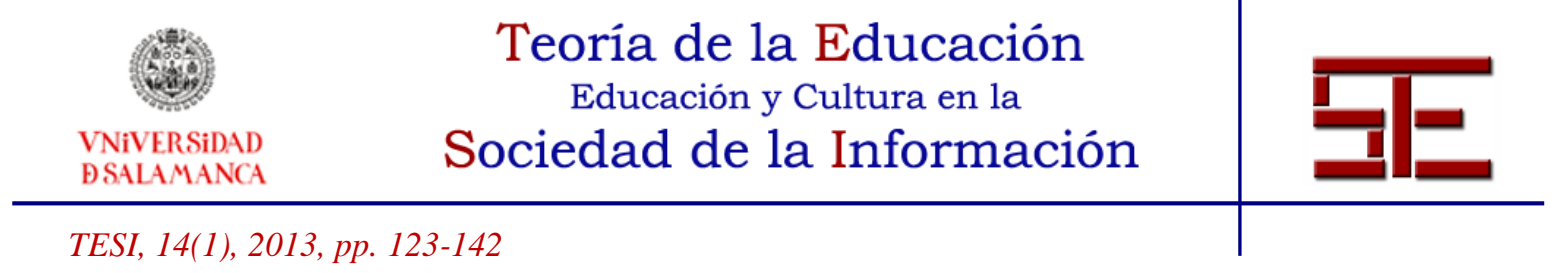

Binkley, M., Erstad, O., Herman, J., Raizen, S., Ripley, M. y Rumble, M. (2010). Defining collaboration skills. Melbourne: The University of Melbourne. Descargado el 15 de noviembre de 2012. http://atc21s.org/.

Bonwell, C. C. y Eison, J. A. (1991). Active Learning: Creating Excitement in the Classroom. ASHE-ERIC Higher Education Report 1. Washington, D.C. George Washington University.

Charsky, D. (2010). From Edutainment to Serious Games: A Change in the Use of Game Characteristics. Games and Culture, 5 (2) 177-198.

Crookall, D., Oxford, R. L. y Saunders, D. (1987). Towards a reconceptualization of simulation: From representation to reality. Simulation/Games for Learning, 17, 147-171.

Csikszentmihalyi, M. (1997). Finding Flow: The Psychology of Engagement with Everyday Life. New York: Basic Books.

Dede, C. (2010). Comparing frameworks for collaboration skills. In J. A. Bellanca, \& R. S. Brandt (Eds.), Collaboration skills: Rethinking how students learn (pp. 51-76). Bloomington: Solution Tree Press.

Fisch, K. \& Mcleod, S. (2009). Did You Know? 3.0-2009 Edition. Descargado el 25 de noviembre de 2012 www.youtube.com/watch?v=PHmwZ96_Gos.

Garris, R., Ahlers, R. y Driskell, J. E. (2002). Games, motivation and learning: a reserach and practice model. Simulations \& Gaming, 33, 441-467.

Guitert, M., Giménez, F., Daradoumis, T. y Marques, J. M. (2000). Virtual Study Groups (VSG), an approach to networked collaborative learning. In J. Bourdeau \& R. Heller (Eds.), Proceedings of World Conference on Educational Multimedia, Hypermedia and Telecommunications 2000 (pp. 382-387). Chesapeake, AACE.

Guitert, M., Guerrero, A., Romeu, T. \& Padros A. (2008). ICT competences for net generation students. International Conference on Advanced Learning Technologies ICALT (IEEE). Santander.

Imaz, J. I. (2011). Pantallas y educación: adolescentes y videojuegos en el País Vasco. Teoria de la Educación. 23(1), 181-200.

Jones, L. K. (1996). Job Skills for the 21st Century: A Guide for Students. Phoenix: Oryx Press.

Malone, T. W. (1981). What makes computer games fun? Byte, 6 (12), 258-277.

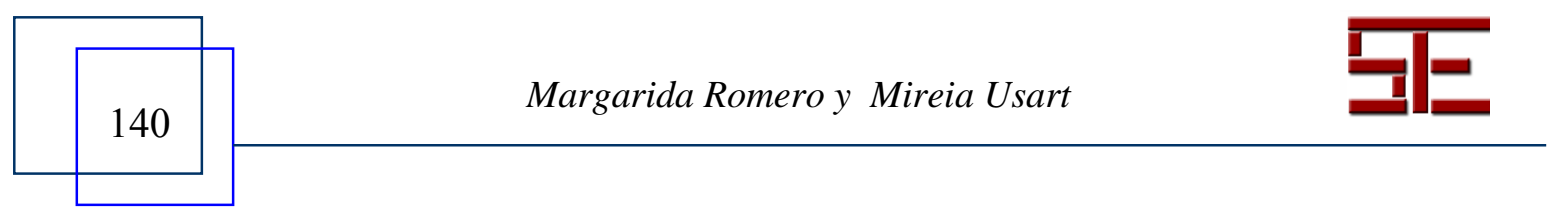




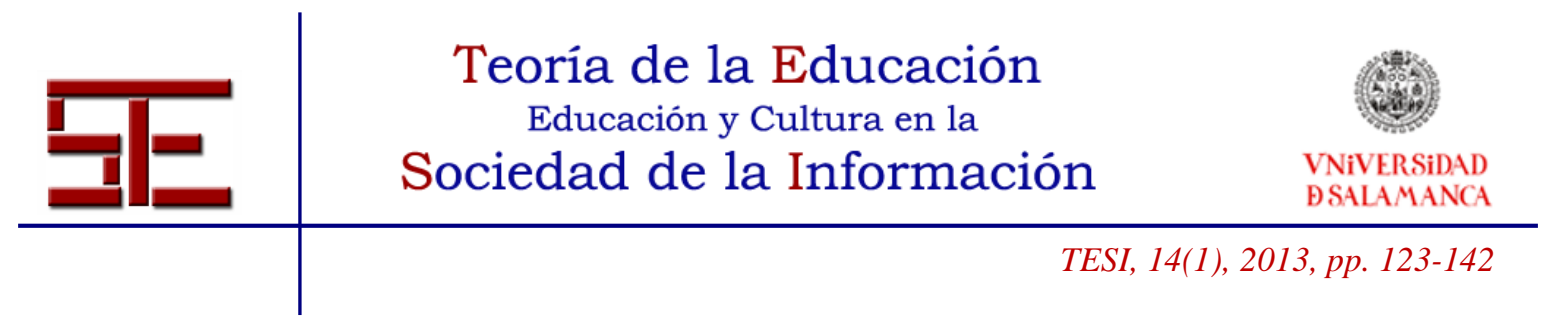

Massons, J., Romero, M., Usart, M., Mas, S., Padrós, A. y Almirall, E. (2011). Uso del aprendizaje basado en juegos en la formación de finanzas para no financieros. Actas de las Jornadas Interuniversitarias de Innovación Docente. Universitat Ramon Llull, DEUSTO, ICADE, 16-17 junio. Barcelona.

Mawdesley, M., Long, G., Al-jibouri, S. y Scott, D. (2011). The enhancement of simulation based learning exercises through formalised reflection, focus groups and group presentation. Computers \& Education, 56, 44-52.

Michael, D. y Chen, S. (2006). Serious Games: Games That Educate, Train and Inform. Boston, Thomson.

Monereo, C. y Pozo, J. I. (2007). Competencias para (con)vivir con el siglo XXI. Cuadernos de Pedagogía, 370, 12-16.

Oblinger, D. (2004). The next generation of educational engagement. Journal of Interactive Media in Education, 1(8), 1-18.

Olmos, S. y Rodríguez, M. J. (2011). Perspectiva tecnológica de la evaluación educativa en la universidad. Teoría de la Educación, 23, 131-157.

Padrós, A., Romero, M. y Usart, M. (2011). Developing serious Games: Form Face-toFace to a Computer-based Modality. E-learning papers, 25, 1-12.

Palloff, R. y Pratt, K. (1999). Building learning communities in cyberspace: Effective strategies for the online classroom. San Francisco: Joseey-Bass.

PRENSKY, M. (2006). "Don't Bother Me Mom -- I'm Learning": How Computer and Video Games Are Preparing Your Kids For 21st Century Success and How You Can Help! Saint Paul (MN): Paragon House.

Redecker, C., Leis, M., Leendertse, M., Punie, Y., Gijsbers, G. y Kirschner, P. (2011). The Future of Learning: Preparing for Change: JRC-IPTS. Descargado el 25 de noviembre de 2012. http://ipts.jrc.ec.europa.eu/publications/pub.cfm?id=4719.

Romero, M., Hyvönen, P. y Barbera, E. (2012). Creativity in collaborative learning across the life span. Creative Education, 3 (4), 422-429.

Romero, M., Usart, M. y Almirall, E. (2011). Serious games in a finance course promoting the knowledge group awareness. EDULEARN11 Proceedings, pp. 3490-3492.

Romero, M., Usart, M., Ott, M., Earp, J., De freitas, S. y Arnab, S. (2012). Learning through playing for or against each other? Promoting collaborative learning in digital

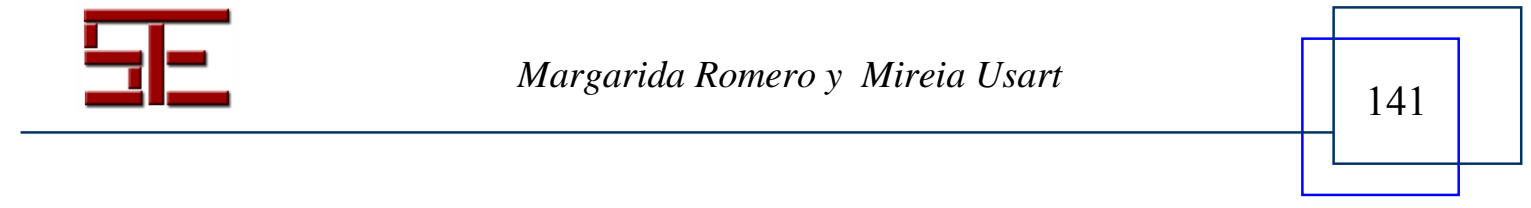




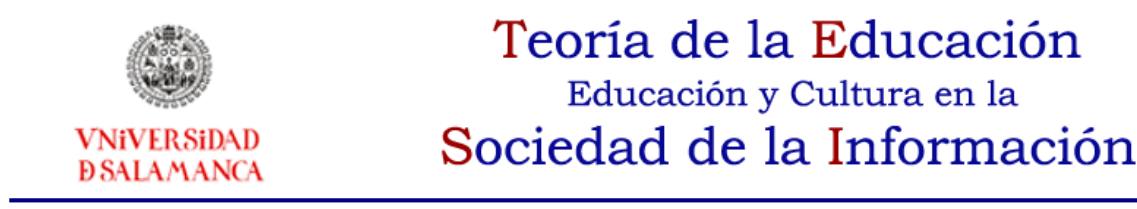

TESI, 14(1), 2013, pp. 123-142

game based learning. 20th European Conference on Information Systems, June 10-13. ESADE, Barcelona.

Romero, M., Usart, M., Popescu, M. y Boyle, E. (2012). Interdisciplinary an international adaption and personalization of the MetaVals Serious Games. The Third International Conference on Serious Games Development and Applications SGDA 2012, 26-29 Sep. University of Bremen, Germany.

Sica, L. S., Delli veneri, A. Y Miglino, O. (2011). Exploring new technological tools for education: Some prototypes and their pragmatical classification. In E learning / Book 1, Elvis Pontes (Eds.) São Paulo, Brazil: Technological Research Institute of São Paulo (IPT).

Stuart, L. y Dahm, E. (1999). 21st centurty skills for 21st century jobs: A report of the U.S. Department of Commerece, U.S. Department of Education, U.S. Department of Labor, National Institute for Literacy and Small Business Administration. Washington, DC.

Thomas, P. y Macredie, R. (1994). Games and the design of human-computer interfaces. Educational and Training Technology International, 31 (2), 134-142.

Trilling, B. y Fadel, C. (2009). Collaboration skills: learning for life in our times. San Francisco, CA: Jossey-Bass.

Usart, M., Romero, M. y Almirall, E. (2011). Impact of the Feeling of Knowledge explicitation in the learners' participation and performance in a collaborative Game Based Learning activity. Proceedings of the International Conference on Serious Games Development and Applications. Springer LNCS.

Voogt, J. y Pareja Roblin, N. (2010). Collaboration skills - Discussion Paper. Enschede (The Netherlands): University of Twente. Descargado el 25 de noviembre de 2012. http://onderzoek.kennisnet.nl/onderzoeken-totaal/21stecentury.

Wittgenstein, L. (1958). The blue and brown books. New York, Harper \& Row.

Para citar el presente artículo puede utilizar la siguiente referencia:

Romero, M. y Usart Rodríguez, M. (2013). Desarrollo de las competencias de colaboración con el uso del Serious Same Metavals. Revista Teoría de la Educación: Educación y Cultura en la Sociedad de la Información. 14(1), 123-142 [Fecha de consulta: $\mathrm{dd} / \mathrm{mm} / \mathrm{aaaa}]$.

http://campus.usal.es/ revistas_trabajo/index.php/revistatesi/article/view/9446/9735

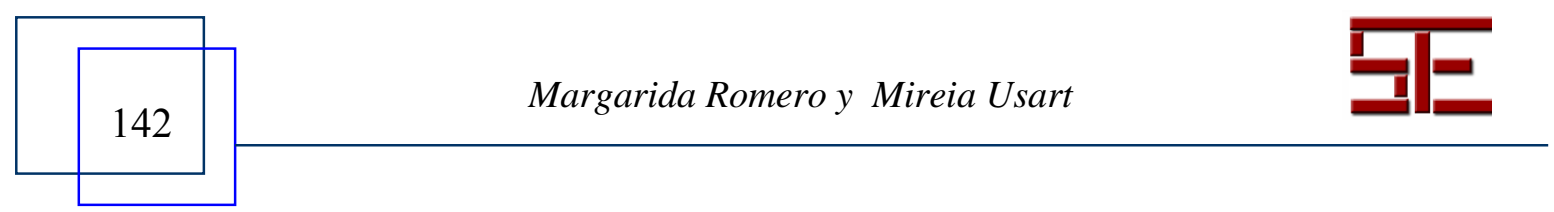

